\title{
Novos pressupostos para o saneamento no controle de arboviroses no Brasil
}

\author{
New premises for sanitation in arbovirus \\ infectious control in Brazil
}

\author{
Nuevas presuposiciones para el saneamiento en \\ el control de arbovirosis en Brasil
}

\author{
Josiane Teresinha Matos de Queiroz ${ }^{1}$ \\ Priscila Neves Silva 1 \\ Léo Heller 1
}

doi: $10.1590 / 0102-311 \times 00223719$

Arboviroses como dengue, Zika e chikungunya vêm causando dor e sofrimento na população e sobrecarregando os sistemas de saúde. Os recentes surtos expuseram a fragilidade das ações de prevenção e controle dessas arboviroses. Portanto, a complexidade das arboviroses ampliou, pois grupos de populações susceptíveis surgem em diferentes áreas geográficas 1,2,3,4,5.

Em 2019, foram notificados 1.544 .987 casos de dengue, 132.205 de chikungunya e 10.768 de Zika. Quanto aos óbitos, foram confirmados 782 por dengue, 92 por chikungunya e três por Zika 6. As altas taxas de incidência de arboviroses transmitidas pelo Aedes nos últimos anos no Brasil podem estar associadas a deficiências no abastecimento de água 7, sejam causadas pelas secas extremas, pela inadequada gestão na prestação de serviços ou pela falta de políticas públicas, e provavelmente pela combinação destes fatores. Ainda assim, podem estar associadas também à inadequação do acesso aos outros componentes do saneamento, como esgotamento sanitário, gestão adequada dos resíduos sólidos e da drenagem de águas pluviais.

Apesar de o país contar com uma legislação que remete a processos de universalização dos serviços de saneamento, conforme o Plano Nacional de Saneamento Básico (PLANSAB), ainda é incipiente a cobertura por abastecimento de água e esgotamento sanitário. Ao se considerar, no planejamento, os pressupostos dos Direitos Humanos à Água e ao Esgotamento Sanitário (DHAES) na elaboração e implementação de políticas públicas, estas seriam mais igualitárias e contribuiriam para a promoção da saúde de populações vulneráveis.

É importante destacar que a forma de organização do território irá definir as relações com o ambiente e, assim, influenciar no surgimento de enfermidades. Com isso, é necessário observar cada território com suas singularidades e localizar o mais precisamente possível onde e como estão acontecendo os agravos, que serviços a população está necessitando, o local de potencial risco sanitário e ambiental e as áreas onde se concentram situações sociais vulneráveis ${ }^{8}$. Nesse contexto, serviços de saneamento podem ser determinantes importantes para a incidência e prevalência de arboviroses e estes têm sido negligenciados nas estratégias de controle das doenças. Ressalta-se que a implementação dos serviços não tem sido orientada pela ótica dos DHAES, que prevê importantes instrumentos analíticos da real situação do território, apontando diretrizes para o planejamento.

Portanto, apresentam-se três premissas para a discussão sobre a relação entre a ocorrência de arboviroses e a oferta de serviços de saneamento, considerando a inclusão dos pressupostos dos DHAES como elemento importante para o controle dessas doenças.
${ }^{1}$ Instituto René Rachou, Fundação Oswaldo Cruz, Belo Horizonte, Brasil.

Correspondência J. T. M. Queiroz Instituto René Rachou, Fundação Oswaldo Cruz. Av. Augusto de Lima 1715 Belo Horizonte, $M G$ 31190-009, Brasil. josiane.matosqueiroz@yahoo. com.br 


\section{Medidas de saneamento e a negligência das dimensões do controle das arboviroses}

O conhecimento da relação entre acesso aos serviços de saneamento e a ocorrência de arboviroses vem se consolidando por pesquisas publicadas no Brasil, como em outros países. Esses estudos vêm identificando que deficiências na oferta de serviços de saneamento têm, em algum grau e para diferentes contextos, relação com a incidência e prevalência dessas doenças. Pesquisas apontam que a ocorrência de dengue reflete a estrutura social de um determinado território, seus fatores de risco e de vulnerabilidade socioambiental. A infestação pelo vetor está presente em diversas localidades, mas a morbidade é maior na população economicamente mais vulnerável, com índices de cobertura precários de saneamento 9,10,11,12,13,14,15,16,17,18,19,20,21,22,23. É importante salientar que as variáveis sanitárias, unicamente, não explicam a heterogeneidade das doenças, pois seus determinantes são múltiplos em aspectos e fatores variados, entretanto, são elementos essenciais para explicar a ocorrência das arboviroses 24,25 .

Para além do conhecimento sobre as questões que afetam a saúde da população, utilizar o território como unidade analítica permite conhecer as possibilidades de intervenção. Com relação ao saneamento, entender e conhecer as práticas culturais e sociais permite identificar as melhores tecnologias a serem utilizadas. As ações de intervenção, quando construídas em âmbito territorial, permitem uma participação ativa da comunidade no processo de tomada de decisão, construindo uma nova forma de governança. As políticas do setor de saneamento geralmente não levam em consideração a situação de saúde das localidades e sua relação com as formas de intervenções e concepções tecnológicas a serem adotadas. Os vieses tecnocêntrico e economicista ainda predominam, e muitas vezes, utilizamse soluções semelhantes em contextos diferentes. Assim, as ações de saneamento podem não alcançar os melhores resultados no que se refere à saúde pública. Exemplificando, a gestão e a manutenção de equipamentos podem ser difíceis para as comunidades mais tradicionais. Abordagens economicistas, que não respeitam as diferenças culturais e sociais, podem não ser efetivas 26,27 .

Como alternativa, valoriza-se o desenvolvimento endógeno de forma participativa e democrática. Salienta-se que o enfrentamento das arboviroses certamente será mais efetivo se os gestores compreenderem a importância dos serviços de saneamento para a promoção da saúde pública, levando em consideração a análise interdisciplinar do território.

\section{Acessos à água e ao esgotamento sanitário e os pressupostos dos DHAES}

O acesso à água foi reconhecido como direito humano em 2010 pela Assembleia Geral, e em 2015 o esgotamento sanitário foi reconhecido como um direito independente, embora integrado ao direito humano à água. Eles derivam de outros direitos, como o direito à saúde física e mental, o direito à vida e à dignidade, estando, portanto, amparados nas leis internacionais de Direitos Humanos 28,29.

Assim, o referencial teórico dos DHAES foi internacionalmente reconhecido de forma explícita e amparado pelas resoluções publicadas anualmente. Essas resoluções implicam obrigações legais aos Estados que devem respeitar, promover e proteger esses direitos, tendo como base pressupostos e princípios como não-discriminação/equidade, sustentabilidade, participação social e democrática, informação, transparência e responsabilização 30,31,32. O acesso à água deve ser fisicamente, geograficamente e financeiramente acessível, e a água deve ser segura, aceitável e estar disponível em quantidade suficiente para uso pessoal e doméstico. Em relação ao esgotamento sanitário, além dos pressupostos referentes ao acesso à água, os países devem assegurar a privacidade do acesso e garantir a dignidade e segurança e ser culturalmente aceitável.

Portanto, a abordagem dos DHAES permite avaliar importantes aspectos para a garantia desses direitos, como os relacionados a gênero, grupos minoritários e vulneráveis, modelos de gestão e aspectos culturais da população, dimensões que vão além meramente das condições da infraestrutura, como a cobertura por redes de canalização 28 .

Neves-Silva \& Heller 29 apontam que o emprego dos DHAES como instrumento para a elaboração de políticas públicas pode favorecer o processo de promoção de saúde da população, pelo reconhecimento dos indivíduos como sujeitos de direito, e a compreensão de que o acesso à água e ao esgotamento sanitário deve ser tratado como direito e não como meramente assistencialismo. 


\section{DHAES e ODS 6 como promotores na proteção da saúde pública}

A quase totalidade dos países pactuou, em 2015, os 17 Objetivos de Desenvolvimento Sustentável (ODS) e suas 169 metas, com a finalidade de erradicar a pobreza, proteger o planeta e assegurar a prosperidade de todos tendo como lema: Ninguém Deve Ser Deixado Para Trás 31 . No que diz respeito ao cumprimento dos DHAES, este está considerado no ODS 6: assegurar a disponibilidade e gestão sustentável da água e saneamento para todas e todos até 2030; eliminar a desigualdade no acesso à água potável, sem discriminação, ter acesso à água segura e de qualidade; e garantir acesso adequado ao esgotamento sanitário visando a acabar com a defecação a céu aberto e dando especial atenção às necessidades das mulheres e daqueles em situação de vulnerabilidade. Assim, o ODS 6 incorpora novos elementos na discussão do conceito de acesso como equidade e a não discriminação, bem como a importância de se ter acesso para além do domicílio, como em escolas e centros de saúde 8 .

O ODS 6 prevê oito metas, abordando o acesso à água e ao esgotamento sanitário e a gestão dos recursos hídricos em uma perspectiva integrada. A preocupação com o fornecimento de água potável é o foco da meta 6.1. A meta 6.2 aborda o esgotamento sanitário, e é interligado às demais metas, pois sabe-se que a falta de coleta e tratamento pode levar à contaminação do solo, mares e fontes de água para abastecimento, prejudicando a saúde pública.

Apesar de os índices de abastecimento de água no país terem apresentado melhoras nas últimas décadas, mesmo com ressalvas de pesquisadores e órgãos oficiais sobre quantidade, qualidade, disponibilidade e acesso, a Agência Nacional das Águas apontou um índice de cobertura em torno de $97,2 \%$ em 2017. Entretanto, ressalta sobre a distribuição do déficit porcentual entre as faixas de renda, sendo a população na faixa de até três salários mínimos a mais afetada pela ausência de abastecimento de água com canalização interna. $\mathrm{O}$ indicador de atendimento no país para o esgotamento sanitário, incluindo rede coletora e fossas sépticas, foi de $63,5 \%$ em 2017, com ressalvas sobre a confiabilidade de dados. Para a universalização da coleta de esgotamento sanitário no país seriam necessários investimentos de aproximadamente 100 bilhões de reais até 2035 e, para o tratamento, foram estimados 47,6 bilhões de Reais. Salienta ainda, que os indicadores, no período histórico avaliado, mostraram avanços, com maiores deficiências nos porcentuais de esgotamento sanitário, enfatizando a necessidade de medidas urgentes. Aborda também sobre diretrizes e recomendações, tendo como horizonte de planejamento o ano de 203533.

O PLANSAB prevê diferentes metas regionais de atendimento para abastecimento de água e esgotamento sanitário até o ano de 2033, buscando atingir a universalização dos serviços. Segundo esse documento, 59,4\% dos domicílios tinham, em 2010, sistema considerado adequado para o abastecimento de água, já para o esgotamento sanitário este percentual era de apenas 39,7\% 34 .

Cabe salientar que, para o cumprimento das metas estabelecidas no ODS 6, é preciso compreender as complexas transformações nos mais diversos ambientes da vida das pessoas, advindos de graves questões relacionadas com os progressivos processos de urbanização, de segregação socioespacial, crises econômicas, incentivos e ações políticas. As situações, sobretudo nas complexas regiões metropolitanas brasileiras, revelam toda a sua carga de exclusão social ou de inclusão precária.

Assim, a elaboração de políticas visando a minimizar a exposição aos vetores das arboviroses e proporcionar ambientes salubres para a população, ao levar em consideração os pressupostos dos DHAES e o território como lócus de atuação, e ainda buscando a equidade e a não-discriminação no acesso ao saneamento a populações vulneráveis, tende a promover resultados mais efetivos no controle das doenças.

\section{Considerações finais}

As arboviroses proporcionam impactos para a saúde pública devido a uma série de fatores, que vão desde a diversidade de agentes infecciosos até a dificuldade na implantação e manutenção de medidas e ações de controle aos vetores. Portanto, ressalta-se a importância do saneamento como forma de controle da incidência e prevalência das arboviroses, considerando a inclusão dos pressupostos dos DHAES no planejamento das ações. 
Este texto destaca que o reconhecimento dos DHAES é um marco relevante para a agenda do setor sanitário, pois apresenta aspectos normativos importantes e urgentes que visam a garantir outras condições, além somente da visão economicista, que ao serem contemplados nas políticas públicas podem alcançar outras parcelas da população.

Dessa forma, valoriza-se a elaboração de políticas e ações integradas, organizadas em conjunto com a sociedade, considerando-se as particularidades e singularidades dos diferentes territórios e privilegiando outros modelos que não sejam aqueles verticalizados, que dificultam a participação efetiva da população e, consequentemente, o enfretamento das arboviroses.

\section{Colaboradores}

J. T. M. Queiroz e P. N. Silva conceberam a ideia original, escreveram e revisaram o manuscrito. L. Heller escreveu e revisou o manuscrito.

\section{Informações adicionais}

ORCID: Josiane Teresinha Matos de Queiroz (00000002-5773-6006); Priscila Neves Silva (0000-00018909-4477); Léo Heller (0000-0003-0175-0180).

\section{Referências}

1. World Helth Organization. Global strategy for dengue prevention and control 2012-2020. https://www.who.int/ denguecontrol/ 9789241504034/en/ (acessado em 19/Jun/ 2018).

2. Weaver SC, Costa F, Garcia-Blanco MA, Ko AI, Ribeiro GS, George S, et al. Zika virus: history, emergence, biology, and prospects for control. Antiviral Res 2016; 130:69-80.

3. Rodriguez-Morales AJ, Villamil-Gomez WE, Franco-Paredes C. The arboviral burden of disease caused by co-circulation and co-infection of dengue, chikungunya and Zika in the Americas. Travel Med Infect Dis 2016; 14:177-9.

4. Barreto ML, Teixeira MG. Dengue no Brasil: situação epidemiológica e contribuições para uma agenda de pesquisa. Estud Av 2008; 22:53-64.

5. Lopes N, Nozawa C, Linhres REC. Características gerais e epidemiologia dos arbovírus emergentes no Brasil. Revista Pan-Amazônica de Saúde 2014; 5:55-64.

6. Secretaria de Vigilância em Saúde, Ministério da Saúde. Monitoramento dos casos de arboviroses urbanas transmitidas pelo Aedes (dengue, chikungunya e Zika), semanas epidemiológicas 01 a 52. Boletim Epidemiológico 2; volume 51. http://portalarquivos2.saude.gov.br/images/ $\mathrm{pdf} / 2020 /$ janeiro/20/Boletim-epidemiologico -SVS-02-1-.pdf (acessado em 20/Jan/2020).

\section{Agradecimentos}

Os autores agradecem à Fundação de Apoio à Fiocruz (Fiotec).
7. Heller L. "Melhoramento dos serviços de água e saneamento é a resposta", apontam especialistas da ONU. http://acnudh.org/pt-br/ho me-2/ (acessado em 06/Jun/2018).

8. World Health Organization; United Nations Children's Fund. Joint monitoring program for water supply and sanitation. Progress on drinking water and sanitation. Update 2017. Geneva: World Health Organization; 2017.

9. Pina MF, Santos SM. Os sistemas de informações geográficas. In: Pina MF, Santos SM, Carvalho MS, organizadores. Conceitos básicos de sistemas de informação geográfica e cartografia aplicados à saúde. Brasília: Organização Pan-Americana da Saúde; 2000. p. 13-4.

10. Vilani RM, Machado JS, Rocha ETS. Saneamento, dengue e demandas sociais na maior favela do Estado do Rio de Janeiro: a Rocinha. Vigil Sanit Debate 2014; 3:18-29.

11. Flausino RF, Souza-Santos R, Oliveira RM. Indicadores socioambientais para vigilância da dengue em nível local. Saúde Soc 2011; 20:225-40.

12. Oliveira RM, Valla VV. Living conditions and life experiences of working-class groups in Rio de Janeiro: rethinking dengue control and popular mobilization. Cad Saúde Pública 2001; 17 Suppl:77-88. 
13. Vilcarromero S, Casanova W, Ampuero JS, Ramal-Asayag C, Siles C, Diaz G, et al. Lecciones aprendidas en el control de Aedes Aegypti para afrontar el dengue y la emergência de Chikungunya en Iquitos, Perú. Rev Peru Med Exp Salud Publica 2015; 32:172-8.

14. Thammapalo S, Chongsuvivatwong AG, Dueravee $M$. Environmental factors and incidence of dengue fever and dengue haemorrhagic fever in an urban area, Southern Thailand. Epidemiol Infect 2008; 136:135-3.

15. Carlton EJ,Liang S, McDowell JZ, Li H, Luo W, Remais JV. Regional disparities in the burden of disease attributable to unsafe water and poor sanitation in China. Bull World Health Organ 2012; 90:578-87.

16. Rey JR, Lounibos LP, Padmanabha H, Mosquera M. Resurgencia del dengue en América: pautas, procesos y prospectos. Interciencia: Revista de Ciencia y Tecnología de América 2010; 35:800-6.

17. Braga C, Luna CF, Martelli CM, Souza WV, Cordeiro MT, Alexander N, et al. Seroprevalence and risk factors for dengue infection in socio-economically distinct areas of Recife, Brazil. Acta Trop 2010; 113:234-40.

18. Teixeira TRA, Medronho RA. Indicadores sócio-demográficos e a epidemia de dengue em 2002 no Estado do Rio de Janeiro, Brasil. Cad Saúde Pública 2008; 24:2160-70.

19. Almeida AS, Medronho RDA, Valencia LI. O. Spatial analysis of dengue and the socioeconomic context of the city of Rio de Janeiro (Southeastern Brazil). Rev Saúde Pública 2009; 43:666-73

20. Lenzi MF, Coura LC, Grault CE, Val MB. Estudo do dengue em área urbana favelizada do Rio de Janeiro: considerações iniciais. Cad Saúde Pública 2000; 16:851-6.

21. Costa JV, Donalisio MR, Silveira LVA. Spatial distribution of dengue incidence and socioenvironmental conditions in Campinas, São Paulo State, Brazil, 2007. Cad Saúde Pública 2013; 29:1522-32.

22. Johansen IC, Carmo RL, Alves LC, Bueno MCD. Environmental and demographic determinants of dengue incidence in Brazil. Rev Salud Pública 2018; 20:346-51.

23. Pedrosa CSG, Souza LRQ, Lima, CVF, Ledur PF, Karmirian K, Gomes TA, et al. The cyanobacterial saxitoxin exacerbates neural cell death and brain malformations induced by Zika vírus. PLoS Negl Trop Dis 2020; 14:e0008060.
24. Buss PM, Pellegrini Filho A. A saúde e seus determinantes sociais. Physis (Rio J.) 2007; 17:77-93.

25. Sommerfeld J, Kroeger A. Eco-bio-social research on dengue in Asia: a multicountry study on ecosystem and community-based approaches for the control of dengue vectors in urban and peri-urban Asia. Pathog Glob Health 2012; 106:428-35.

26. Aleixo B, Pena JL, Heller L, Rezende S. Infrastructure is a necessary but insufficient condition to eliminate inequalities in access to water: research of a rural community intervention in Northeast Brazil. Sci Total Environ 2019; 652:1445-55.

27. Silva PN, Cabral AR, Dias AP, Matida AH, Kligerman DC, Carneiro FF, et al. Saneamento e saúde. Saneamento: entre os direitos humanos, a justiça ambiental e a promoção da saúde. Rio de Janeiro: Editora Fiocruz; 2018. (Série Fiocruz. Documentos Institucionais) (Coleção Saúde, Ambiente e Sustentabilidade, 6).

28. Brown C, Neves-Silva P, Heller L. The human right to water and sanitation: a new perspective for public policies. Ciênc Saúde Colet 2016; 21:661-70.

29. Neves-Silva P, Heller L. O direito humano à água e ao esgotamento sanitário como instrumento para promoção da saúde de populações vulneráveis. Ciênc Saúde Colet 2016; 21:1861-9.

30. United Nations General Assembly. Human right to water and sanitation. Geneva: United Nations General Assembly; 2010. (UN Document A/RES/64/292).

31. United Nations. Resolution adopted by the General Assembly on 25 September 2015. Transforming our world: the 2030 Agenda for Sustainable Development. Geneva: United Nations; 2015.

32. United Nations Human Right Council. Report of the special rapporteur on the human right to safe drinking water and sanitation. Geneva: United Nations Human Right Council; 2015.

33. Agência Nacional de Águas. ODS 6 no Brasil: visão da ANA sobre os indicadores. https:// www.ana.gov.br/acesso-a-informacao/insti tucional/publicacoes/ods6/ods6.pdf (acessado em 18/Dez/2019).

34. Secretaria Nacional de Saneamento Ambiental, Ministério das Cidades. Plano Nacional de Saneamento Básico. http://www.cidades.gov. $\mathrm{br} /$ images/stories/ArquivosSNSA/PlanSaB/ plansab_texto_editado_para_download.pdf (acessado em 23/Mai/2018).
Recebido em 06/Dez/2019

Versão final reapresenatda em 10/Fev/2020

Aprovado em 14/Fev/2020 